\title{
REVIEW ARTICLE OPEN Pathogenesis and multidisciplinary management of medication-related osteonecrosis of the jaw
}

\author{
Lina $\mathrm{He}^{1,2}$, Xiangyu Sun ${ }^{1,2}$, Zhijie Liu ${ }^{1,2}$, Yanfen Qiu ${ }^{1,2}$ and Yumei Niu (iD)
}

\begin{abstract}
Medication-related osteonecrosis of the jaw (MRONJ) is a serious side effect of bone-modifying agents and inhibits angiogenesis agents. Although the pathogenesis of MRONJ is not entirely clear, multiple factors may be involved in specific microenvironments. The TGF- $\beta 1$ signalling pathway may have a key role in the development of MRONJ. According to the clinical stage, multiple variables should be considered when selecting the most appropriate treatment. Therefore, the prevention and management of treatment of MRONJ should be conducted in patient-centred multidisciplinary team collaborative networks with oncologists, dentists and dental specialists. This would comprise a closed responsibility treatment loop with all benefits directed to the patient. Thus, in the present review, we aimed to summarise the pathogenesis, risk factors, imaging features, clinical staging, therapeutic methods, prevention and treatment strategies associated with MRONJ, which may provide a reference that can inform preventive strategies and improve the quality of life for patients in the future.
\end{abstract}

International Journal of Oral Science (2020)12:30

; https://doi.org/10.1038/s41368-020-00093-2

\section{INTRODUCTION}

Bisphosphonates (BPs) are the most widely used antiresorptive drugs in the management of cancer-related conditions, such as the prevention of bone metastatic malignancies, and are also used for the treatment and prevention of osteoporosis. However, since the reporting of the first case of osteonecrosis of the jaw (ONJ) in cancer patients who had been treated with high-dose BPs in $2003,{ }^{1}$ it has been acknowledged that bisphosphonate-related osteonecrosis of the jaw (BRONJ) is a serious adverse reaction to $B P s$. In response to the incidence of adverse reactions to another bone-modifying agent (BMA), denosumab, or the angiogenesis inhibitor agent bevacizumab, the American Association of Oral and Maxillofacial Surgeons (AAOMS) renamed BRONJ 'medicationrelated osteonecrosis of the jaw' (MRONJ), ${ }^{2}$ as described in a key paper (2014) from the AAOMS.

Drugs that cause MRONJ are grouped into two categories: BPs, used for osteoporosis or malignancy, and non-BPs, including other antiangiogenic or antiresorptive medications. Therapeutic indications, the type of medication and the mode and duration of administration of BPs or antiresorptive therapy are related to the occurrence of MRONJ. The risk of using injectable BPs in patients with malignant tumours is significantly higher when using oral BPs for patients with osteoporosis. ${ }^{3}$ An association with MRONJ has been observed in $\sim 1 \%$ to $9 \%$ of patients with advanced cancer who are prescribed injectable BMAs. ${ }^{4}$ For patients receiving oral BPs for osteoporosis, the prevalence of ONJ is $\sim 0.21 \%$ after at least 4 years of exposure to BPs. ${ }^{5}$

A diagnosis of MRONJ should be considered when patients present with all three of the following criteria: (1) previous or current treatment with a BMA or angiogenesis inhibitor; (2) exposed bone or bone that can be probed through an intraoral or extraoral fistula in the maxillofacial region that has persisted for longer than 8 weeks; and (3) no history of radiation therapy of the jaws or metastatic disease of the jaws. ${ }^{2}$ A pathological diagnosis is helpful for the diagnosis of MRONJ. The most commonly observed pathology is the exposure of bone not covered with epithelium, a reduced number of osteocytes, increased quantities of necrotic bone with a greater number of empty lacunae, demineralised extracellular bone matrix, denudation of the bone and osteonecrosis. ${ }^{6}$ The differential diagnosis of MRONJ should exclude atypical neuralgias, odontalgia, dental caries, pulpitis, periapical pathologies, periodontal diseases, myofascial pain, sinusitis, fibroosseous lesions, neoplastic processes in the jaw, chronic sclerosing osteomyelitis, alveolar osteitis, sarcomas, or temporomandibular joint disorders. In rare situations, osteoradionecrosis should be strongly considered in patients with an exposed bone who have received treatment with BPs and radiation therapy to the jaw. Inflammation and infections of the bone, with clinical symptoms similar to those of osteomyelitis, are typical secondary events. Furthermore, for patients at risk of or with established MRONJ that have other common clinical conditions, symptoms should not be confused with MRONJ.?

\section{PATHOGENESIS AND RISK FACTORS OF MRONJ}

Although the pathogenesis of MRONJ has not been entirely clarified, several hypotheses have been suggested.

\section{Unique characteristics of the jaw}

Although BPs affect the function of osteoclasts within the skeletal system, only the jaw can suffer from osteonecrosis. The oral cavity has a number of unique characteristics that make it a distinctive environment. The mandible is high in calcium, which may absorb a greater quantity of BPs than the long bones. ${ }^{8}$ Furthermore, the

\footnotetext{
${ }^{1}$ The First Affiliated Hospital of Harbin Medical University, Harbin, China and ${ }^{2}$ School of Stomatology, Harbin Medical University, Harbin, China Correspondence: Yumei Niu (niuym@hrbmu.edu.cn)

These authors contributed equally: Lina He, Xiangyu Sun
}

Received: 4 March 2020 Revised: 17 August 2020 Accepted: 18 August 2020

Published online: 21 October 2020 
long bones are produced by endochondral ossification, while the mandibles develop principally through intramembrane ossification. Human mandibles have been shown to contain more collagen than long bones. ${ }^{8}$ These anatomical characteristics ensure that the jaw bones are unique, with a propensity to suffer osteonecrosis. The close relationship between the teeth and jaw bone provides a route for microorganisms and other inflammatory agents to enter the bone, a situation not found in any other anatomical location. Dentoalveolar surgery is considered a major risk factor for MRONJ, especially tooth extraction. ${ }^{9}$ The placement of dental implants and endodontic or periodontal surgery requiring exposure and manipulation of bone are also risk factors.

BPs have a greater effect on the cells of the craniofacial bones than those of the ilium and tibia. Mandibular mesenchymal stem cells have demonstrated a higher proliferation rate than long bone mesenchymal stem cells. ${ }^{10}$ It has been reported that the migration of dental stem cells localised in close proximity to the jaw bone decreases following the administration of BPs. ${ }^{11}$ A recent report suggested that BPs can induce the production of reactive oxygen species, which inhibit the proliferation and migration of oral fibroblasts, thereby contributing to the pathogenesis of BRONJ. ${ }^{12} \mathrm{Li}$ et al. $^{13}$ found that cell proliferation, adhesion, migration and osteogenic differentiation of periodontal ligament stem cells decreased significantly as a result of BRONJ lesions, a factor possibly important in the underlying mechanisms of BRONJ.

Altered balance of osteoblasts and osteoclasts in bone remodelling

Bone remodelling is initiated by osteoclast-mediated bone resorption, in which the absorbed bone is replaced by fresh bone tissue produced by osteoblasts. BPs increase osteoclast apoptosis and other antiresorptive drugs inhibit osteoclast differentiation and function, resulting in decreased bone resorption and remodelling. The long bones contain a greater quantity of bone marrow fat than flat bones, and murine long bones contain more osteoclast precursors than jaw bones. ${ }^{14}$ Osteoclasts in the jaw are more sensitive to BPs than those in the long bones. ${ }^{15}$ If the accumulation of BPs within a bone reaches a toxic level, the BPs can affect the survival of osteoblasts and their progenitor cells.

Wehrhan et al. ${ }^{16}$ first demonstrated that TGF- $\beta 1$ signalling participates in MRONJ. It has an important role in bone remodelling through enhanced matrix production and osteoblast differentiation. Smad $2 / 3$ has been identified as a downstream effector of TGF- $\beta 1$. A recent study demonstrated that treatment with $\mathrm{BPs}$ reduces the expression of BMP-2, which has a major role in bone remodelling, development and osteoblast differentiation. Early differentiation marker type I collagen, intermediate differentiation markers, such as Osterix and alkaline phosphatase (ALP), and the late differentiation marker Osteocalcin has been shown to be suppressed by TGF- $\beta 1$ combined with low doses of BPs in osteoblasts. In particular, Runx-2 is regulated through Smad 2/3. ${ }^{17}$

Furthermore, TGF- $\beta 1$ is involved in the synthesis of RANKL through the reduced ability of osteoblasts to secrete RANKL, which stimulates osteoclasts via its receptor, RANK. It has been reported that the treatment of osteoblasts with BPs increases the expression of TGF- $\beta 1$, resulting in reduced expression of RANKL. OPG, a soluble protein produced by osteoblasts, can inhibit the interaction between RANKL and RANK. ${ }^{17,18}$ BP treatment may alter the RANKL-OPG complex. ${ }^{17}$ The RANK/RANKL/OPG signalling pathway is triggered in MRONJ subjects. ${ }^{19} \mathrm{~A}$ recent study demonstrated that zoledronate can enhance osteoclastogenesis through elevated expression of interleukin-6 (IL-6), followed by activation of the STAT3 pathway, which is related to vessel regeneration around bone tissues, ${ }^{20}$ and finally the expression of RANKL. ${ }^{21}$ Denosumab, a recently developed antiresorptive medication, is an anti-RANKL antibody that utilises the same mechanism of action as OPG. ${ }^{22}$ By blocking RANKL/RANK interaction, denosumab decreases bone resorption. Using different antiresorptive mechanisms, both BPs and denosumab inhibit osteoclasts and decrease the rate of bone turnover.

Given that the expression of RANKL is altered by multiple signalling pathways, the ratio of osteoblasts to osteoclasts in bone remodelling becomes altered, reducing bone resorption and turnover and giving rise to the accumulation of non-renewed and hypermineralized bone. Changes in the microenvironment of the periosteum cannot provide sufficient nutrition for the jaw, so osteonecrosis occurs following changes in the external environment. Therefore, although bone remodelling representing the pathogenesis of MRONJ may be regulated by multiple signalling pathways, the specific regulatory mechanism should be investigated further.

\section{Infection and Immunity}

Infection or inflammation has long been considered a critical factor in the pathogenesis of ONJ. Bacteria have been found in biopsied specimens of necrotic bone removed from patients with ONJ. ${ }^{23}$ Pre-existing dental or periodontal infection in patients treated with antiresorptive or antiangiogenic medications increases the risk of MRONJ. ${ }^{24}$ Extraction of teeth with serious periodontal or periapical infections is a risk factor for the development of MRONJ.25 Another study found that periapical and periodontal infections both with and without tooth extraction can increase the risk of MRONJ because the infection is responsible for modifying the number and function of osteoclasts. ${ }^{26}$ Furthermore, local changes in $\mathrm{pH}$ caused by dentoalveolar infection or surgery are the principal factors responsible for the development of BRONJ. ${ }^{27}$ Therefore, inflammatory oral disease is a recognised risk factor for the development of MRONJ. Periodontal or periapical diseases are considered relevant for MRONJ. ${ }^{28}$ Through extensive oral health controls that prevent oral infections, the incidence of MRONJ can be significantly reduced. ${ }^{7}$ The proinflammatory cytokine IL-36 has been found to be present in the gingival crevicular fluid in periodontal diseases. Notably, IL$36 a$ is highly upregulated in MRONJ lesions and has an aetiological role in the development of MRONJ. Importantly, it has been demonstrated that there is crosstalk between the IL-36a and TGF- $\beta$ signalling pathways, ${ }^{29}$ suggesting that infection or inflammation are key factors in the pathogenesis of MRONJ, at least in part through the TGF- $\beta$ signalling pathways.

The immune system is closely related to bone loss and bone regeneration. Representing innate lymphocytes, gamma delta $\mathrm{T}$ cells are important in bone regeneration. Such $\mathrm{T}$ cells are significantly reduced in osteoporotic patients who are treated with BPs, indicating that a connection exists between MRONJ and gamma delta $T$ cell deficiency. ${ }^{30}$ Neutrophils promote wound healing following noninfective injury. Nitrogen-containing BPs alter the defence capabilities of neutrophils and impair normal wound healing, possibly representing a critical role in the pathogenesis of MRONJ. ${ }^{31}$ Macrophages are sensitive to BPs, which cause an inhibitory effect and reduce the viability and differentiation capability of the macrophages. The function of macrophages is disrupted by increased MMP expression, leading to impaired wound healing in MRONJ-affected areas. ${ }^{32}$ Through the inhibition of RANKL, denosumab may affect the expression of RANK on immune cells, such as dendritic cells, monocytes, or macrophages. RANKL increases the production of proinflammatory cytokines and reduces monocyte apoptosis. Thus, denosumab inhibits the RANK-RANKL interaction, resulting in MRONJ, which may be related to a change in the function and survival of monocytes and macrophages. ${ }^{33}$ Therefore, as weak evidence, both BPs and denosumab might facilitate infection of the bone surface, resulting in an increased risk of MRONJ. Interleukins, proteins produced by immune cells, are related to the expression and regulation of the immune response, which is involved in multiple factors from lymphocytes and macrophages. It is noteworthy that IL- 6 and IL-36a expression are elevated following 
treatment with BPs. ${ }^{29}$ IL-6 subsequently activates the STAT3 pathway, while IL-36a activates the ERK signalling pathway and subsequently inhibits translocation of TGF- $\beta 1$ and the Smad signalling pathway. ${ }^{21,29}$ Furthermore, TLR-4-mediated macrophage polarisation participates in the pathogenesis of BRONJ in mice. ${ }^{34}$ Therefore, it is possible that multiple signalling pathways participate in the pathogenesis of MRONJ.

\section{Angiogenesis}

Vascular endothelial growth may be a critical factor in the pathogenesis of MRONJ. ${ }^{35}$ Zoledronate has direct inhibitory effects on angiogenesis and vascular damage, possibly contributing to the development of MRONJ in its users, owing to reduced angiogenesis impairing healing after the intervention. ${ }^{36}$ The antiangiogenic effects of denosumab and zoledronate were compared, and the findings suggest that zoledronate exhibits negative effects on angiogenesis, while denosumab may not have antiangiogenic activity. ${ }^{37}$ Vascular endothelial growth factor (VEGF) has an essential role in angiogenesis. The antiangiogenic properties of BPs are directly linked to the pathogenesis of MRONJ, and serum VEGF levels could represent an effective early predictive marker. $^{35}$ Monoclonal antibodies targeting VEGF receptors, ${ }^{38}$ as antiangiogenic drugs, are prescribed in cancer patients to prevent metastasis through the blood and lymph nodes, resulting in ischaemia and eventually MRONJ. ${ }^{39}$ VEGF synthesis is stimulated by TGF- $\beta .^{40}$ The expression of TGF- $\beta$ and angiogenesis-related signalling have been shown to be possible consequences of MRONJ. ${ }^{41}$ Altered VEGF expression has been observed following treatment with $\mathrm{BPs}^{42}$ possibly related to the expression of TGF- $\beta .^{16}$

\section{Soft tissue toxicity}

Although osteoclasts and bone are the primary targets following their exposure to BPs, it has been reported that the toxicity of BPs to soft tissue is closely related to MRONJ. Mucosal ulcerations may be the initial pathologic event that occurs in MRONJ. ${ }^{43}$ BPs increase apoptosis and decrease proliferation in a number of cell types in vitro. ${ }^{12,13}$ In addition, the administration of zoledronic acid to oral gingival fibroblasts in vitro has been found to reduce the expression of extracellular matrix (ECM) proteins, including collagens I, II and III. ${ }^{44}$ It has been shown that impairment of TGF$\beta 1$ signalling is related to oral mucosal soft tissue repair in BRONJ. ${ }^{16}$ Increased TGF- $\beta 1$ and Smad $2 / 3$ expression are related to fibrocontractive wound healing disorders. ${ }^{45}$ Alterations in TGFB1 signalling after BP treatment might explain BP-associated changes in the oral mucosal tissues of MRONJ.

\section{Other factors}

Systemic diseases may increase the risk of MRONJ. BPs are occasionally administered to patients with rheumatoid arthritis to reduce bone destruction and control osteoporosis. ${ }^{46}$ However, BPs have been found to be associated with MRONJ in such patients, especially following their use over long durations and in high doses. Administration of zoledronate, among the most common BPs used clinically, has been shown to result in more serious MRONJ in experimental mice with rheumatoid arthritis. ${ }^{47}$ Therefore, rheumatoid arthritis may be a risk factor for the pathogenesis of MRONJ. Diabetes mellitus promotes inflammation and induces a change in the function of immune cells, which may affect the pathogenesis of MRONJ. It has been reported that diabetic mice are more likely to suffer from MRONJ. The relationship between diabetes mellitus and the pathogenesis of MRONJ is also related to other pathways of injury, such as microvascular ischaemia and reduced bone remodelling. ${ }^{48}$

Genetic factors have a moderate effect on the occurrence of MRONJ. There is an association between the presence of one or more single nucleotide polymorphisms (SNPs) and the appearance of MRONJ. ${ }^{49}$ The majority of SNPs are located in regions of genes associated with bone turnover or certain metabolic bone diseases. In addition, there may be germline sensitivity to BPs. ${ }^{50}$ Corticosteroids also increase the risk of MRONJ.9 Age, gender, tobacco use and type of cancer are variable risk factors for MRONJ. ${ }^{51-53}$

Although the hypotheses above have been developed to elucidate the pathogenesis of and risk factors for MRONJ, the mechanisms are not yet entirely clear. TGF- $\beta 1$ signalling, a relatively well-elucidated pathway closely related to the pathogenesis of MRONJ, may have a key role in the development of MRONJ (Fig. 1). Additional mechanisms underlying the pathophysiology of MRONJ remain to be elucidated.

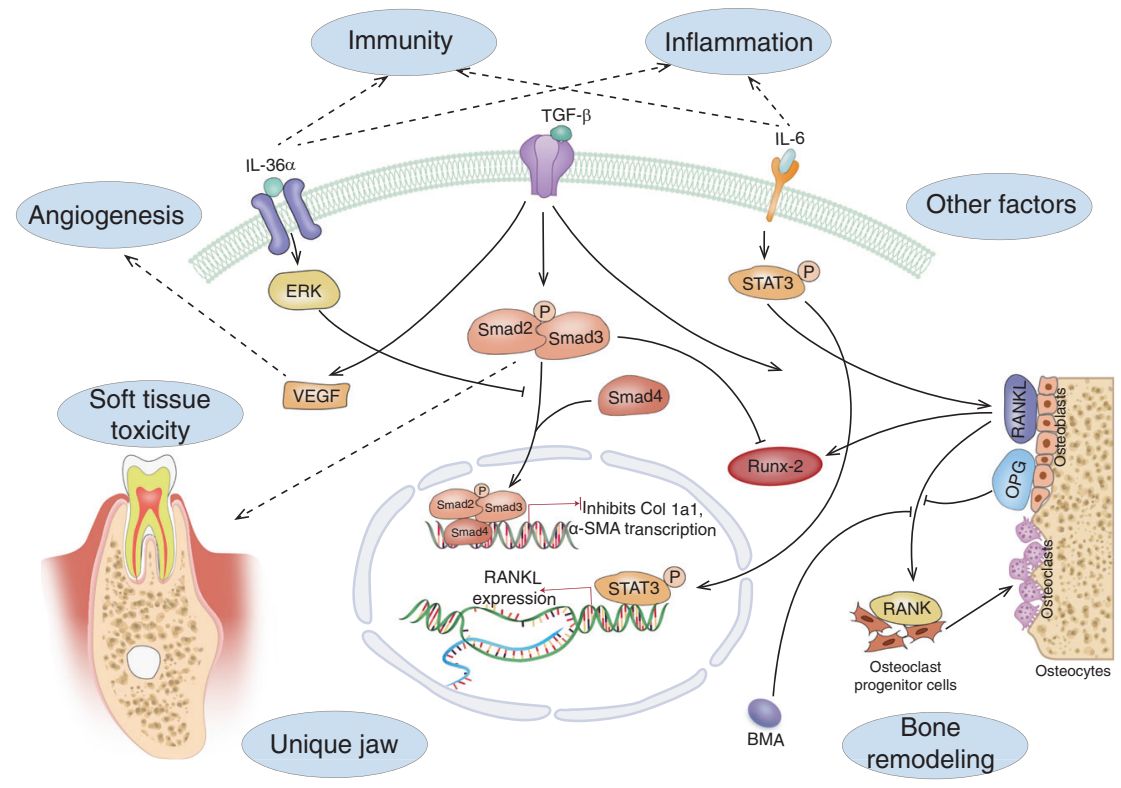

Fig. 1 The hypotheses of MRONJ pathogenesis. 
Table 1. Clinical conditions and Imaging features by stage of MRONJ according to the American Association of Oral and Maxillofacial Surgeons

\begin{tabular}{|c|c|c|}
\hline MRONJ stage & Clinical conditions & Imaging features \\
\hline At risk & $\begin{array}{l}\text { No apparent necrotic bone in patients treated with bone- } \\
\text { modifying agents }\end{array}$ & Nonspecific radiographic changes \\
\hline Stage 0 & $\begin{array}{l}\text { No clinical evidence of necrotic bone, but nonspecific clinical } \\
\text { findings and symptoms }\end{array}$ & $\begin{array}{l}\text { Alveolar bone loss or resorption } \\
\text { Clerotic alveolar bone, thickening and sclerosis of the lamina dura } \\
\text { Thickening or obscuring of the periodontal ligament }\end{array}$ \\
\hline Stage 1 & $\begin{array}{l}\text { Exposed and necrotic bone or fistulas that probe to the bone in } \\
\text { patients who are asymptomatic and have no evidence of infection }\end{array}$ & $\begin{array}{l}\text { May present same as stage } 0 \\
\text { Changes to trabecular pattern: disorganised, trabecular pattern } \\
\text { and poor corticomedullary differentiation }\end{array}$ \\
\hline Stage 2 & $\begin{array}{l}\text { Exposed and necrotic bone in patients with pain and clinical } \\
\text { evidence of infection }\end{array}$ & $\begin{array}{l}\text { Mixed diffuse osteosclerosis, osteolysis from the alveolar bone to } \\
\text { the jaw bone, thickening of the mandibular canal, periosteal } \\
\text { response, maxillary sinusitis and sequestration }\end{array}$ \\
\hline Stage 3 & $\begin{array}{l}\text { Exposed and necrotic bone or a fistula that probes to the bone in } \\
\text { patients with pain, infection and one or more of the following: } \\
\text { exposed and necrotic bone extending beyond the region of } \\
\text { alveolar bone resulting in pathologic fracture, extraoral fistula, oral } \\
\text { antral or oral-nasal communication or osteolysis extending to the } \\
\text { inferior border of the mandible or sinus floor }\end{array}$ & $\begin{array}{l}\text { Osteosclerosis/osteolysis of the surrounding bone, pathologic } \\
\text { mandibular fracture and osteolysis extending to the maxillary } \\
\text { sinus floor }\end{array}$ \\
\hline
\end{tabular}

\section{CLINICAL STAGING AND IMAGING FEATURES OF MRONJ}

A clinical staging system was used to categorise patients with MRONJ. Patients with no apparent necrotic bone are considered to be 'at-risk' if they have been treated with bone-modifying agents. Stage 0 patients have no clinical evidence of necrotic bone but present with nonspecific symptoms or clinical and radiographic findings. Stage 1 is defined as patients having exposed and necrotic bone or fistulas that probe to the bone and who are asymptomatic and have no evidence of infection. Stage 2 is defined as individuals with exposed and necrotic bone and who have pain and clinical evidence of infection. Stage 3 is defined as patients with exposed and necrotic bone or fistulas that probe to bone with evidence of infection and at least one defined characteristic. According to the AAOMS, imaging is critical in the diagnosis and assessment of disease progression in MRONJ patients. ${ }^{27}$ Orthopantomography, CT scanning and MRI are most commonly used to diagnose MRONJ. A recent study demonstrated that the qualitative assessment of MRONJ with ultrashort echotime (UTE) magnetic resonance (MR) imaging was comparable to the reference standard cone-beam computed tomography $(\mathrm{CBCT}){ }^{54}$ Bone scintigraphy contributes to the early detection of MRONJ in high-risk patients. ${ }^{55}$ In animals with MRONJ, the most consistent macroscopic findings are necrosis, denuded bone and formation of fistula and pus. . $^{6}$ Arce et al. assessed patients with MRONJ using six techniques, including films and magnetic resonance imaging. The imaging findings included osteosclerosis, osteolysis, dense woven bone, thickened lamina dura, subperiosteal bone deposition and failure of postsurgical remodelling. ${ }^{56}$ Imaging findings are different for different clinical stages (Table 1 and Fig. 2), but the definition of MRONJ does not currently include imaging-related criteria. ${ }^{4}$ Compared with typical imaging changes in stages 2 and 3, such as large-scale necrotic bone separation or pathological fracture, more attention should be paid to changes observed in the imaging of patients at stage 0 . The capture of subtle changes in bone (as shown in Fig. 2a), which are not easy to detect, may have an important role in the follow-up treatment to delay the progression of the disease.

\section{PREVENTION AND MDT TREATMENT STRATEGIES FOR MRONJ}

Multiple variables, including age, sex, disease status, MRONJ stage and lesion size, ${ }^{7}$ should be considered prior to the assessment of risk and selection of a treatment plan. Therefore, personalised treatment plans and multidisciplinary collaborative therapy should be used clinically. The collaboration of oncologists, dentists and dental specialists should be practised at every period of treatment, sharing treatment information contemporaneously.

The key to the prevention and treatment of MRONJ is its detection and diagnosis at an early stage to avoid the risk of progression and effectively prevent its occurrence by screening high-risk patients who are prone to the disease (e.g. AAOMS stage $0)^{4}$ Therefore, prevention should precede treatment. Published studies have suggested that oral hygiene and treatment of local infections can reduce the risk of MRONJ. ${ }^{57-59}$ Close follow-up and multidisciplinary collaborations between dentists and oncologists have a vital role in the prevention and treatment of MRONJ. A multidisciplinary collaboration system should be established.

More attention should be paid to oral care and periodontal and general health education for all stages of MRONJ. ${ }^{60}$ Treatment strategies for the category include the treatment of patients with modifiable risk factors and the management of avoidable risk factors. Maintenance of meticulous oral hygiene and the use of antibacterial mouth rinses can help delay the progression of MRONJ. ${ }^{4}$

Cancer patients who plan to receive BMA in non-emergency situations should be evaluated for oral care prior to the start of treatment to ensure that the necessary medical dental procedures are performed before the start of BMA. ${ }^{4}$ Dental follow-up should be conducted using a routine schedule, such as every 6 months following the start of BMA treatment, ${ }^{7,61}$ but a clear plan for monitoring the progress of the disease has not been put forward in detail. ${ }^{62}$ Multidisciplinary team members should work with patients at an early stage to address the risk factors for MRONJ. Selective alveolar procedures should not be performed during aggressive treatment with BMA at tumour doses. ${ }^{63}$

For prevention and the process management of MRONJ, multidisciplinary experts and patients themselves should be involved to improve the process, ${ }^{4}$ performing their individual roles (Table 2). Oncologists should inform patients about the importance of oral care prior to treatment and that they should undergo an examination by a dentist to eliminate, as far as possible, any risks. For patients recently diagnosed with MRONJ, whether BMA therapy should be continued or discontinued can be ascertained, determining the development of subsequent treatment plans. Modifiable risk factors should be reinforced: invasive dental procedures, diabetes, periodontal disease, denture use and smoking. The dentist should also be provided with information about the patient's medical diagnosis and antiabsorption and angiogenesis inhibitors used to indicate whether the patient had already commenced therapy and its duration. ${ }^{64}$ 
a
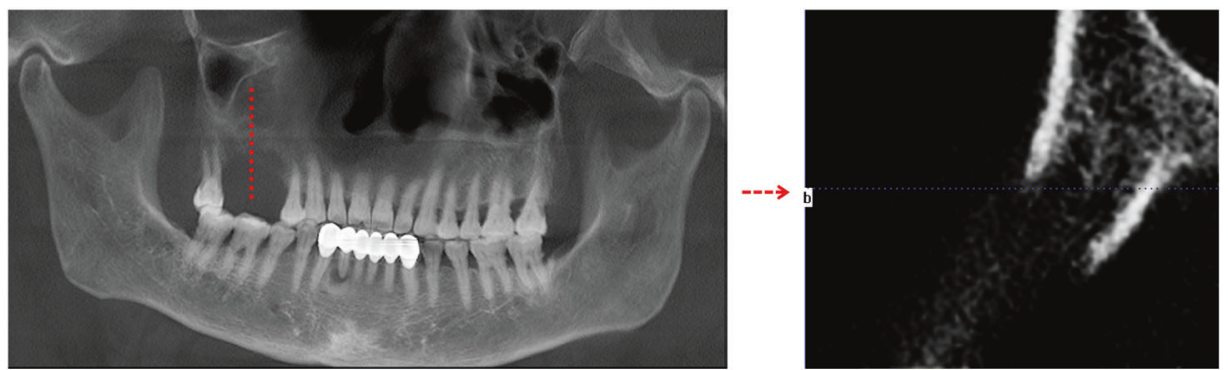

b
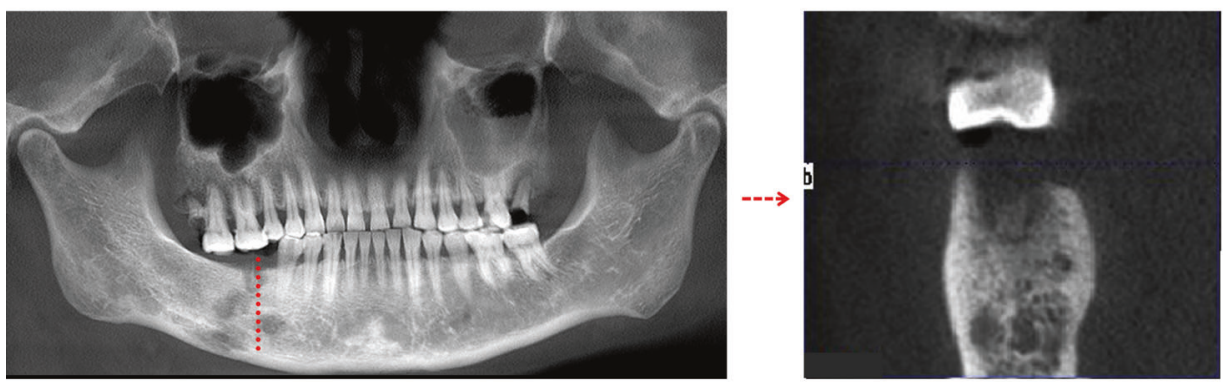

C
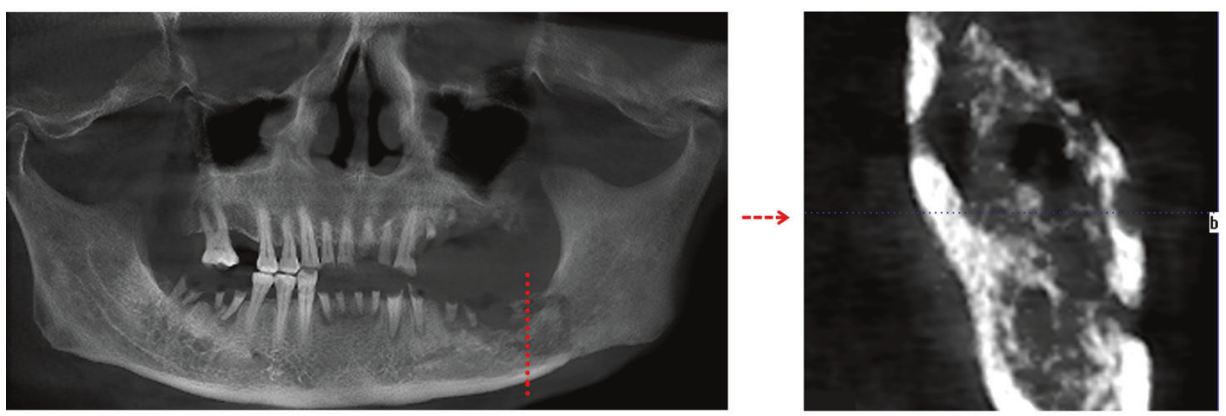

d
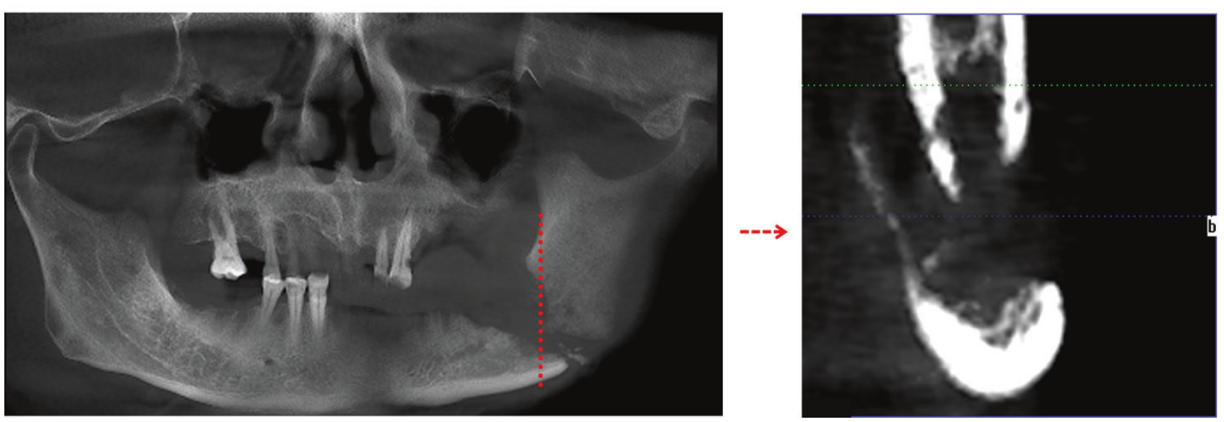

Fig. 2 Imaging findings in different clinical stages.

For dentists, treatments should be consistent, with a follow-up system established that ensures follow-up examinations. For patients prior to cancer treatment, modifiable risk factors should be evaluated, and the required pre-treatments should be improved: complete dental examinations, including orthopaedic photography and intraoral radiography, required extractions, conservative dental and periodontal treatments, adjustment of prosthetics if required, and finally education about the requirement for a lifelong daily commitment to oral care and encouraging the correction of risk factors (such as smoking ${ }^{65}$ and uncontrolled diabetes ${ }^{66,67}$ ). Controllable risk factors should be minimised. During the course of treatment, follow-up for 6 months should be strictly adhered to, and the doctor should be consulted at any time if symptoms reappear. It is recommended that the dentist complete an oral examination, evaluate the status of the soft and hard tissues in the oral cavity, continue oral education and control risk factors. Patients should ensure that they communicate with their oncologist at any time. ${ }^{68-70}$
When a suspected MRONJ patient consults a dentist or oncologist, the patient should be referred to a dental specialist for additional treatment management. ${ }^{71}$ The dental specialist should evaluate the clinical stage, the severity of the disease and symptoms, functional impact and overall prognosis based on clinical manifestations following an 8-week follow-up plan. In accordance with the staging of MRONJ, the dental specialist should design a treatment plan for its management and report that plan to the oncologist. The patient should be followed-up for 8 weeks, the outcome of which should be assessed by a dental specialist. $^{72,73}$

Prevention and treatment management of MRONJ should be patient-centred through an MDT collaborative network with oncologists, dentists and dental specialists. Each treatment team should be responsible for each stage of the patient's progress to complete the corresponding tasks (Table 3). The treatment team also needs to share treatment resources and be able to communicate at any time to develop a follow-up treatment 
Table 2. Division of tasks for multidisciplinary management of MRONJ

Participating disciplines Task division

Oncology 1. Determine the continuation or discontinuation of BMA therapy in patients

2. Refer patient for dental assessments and need for commitment to oral care

3. Reinforce modifiable risk factors

4. Provide the dentist with the patients' medical diagnosis and antiresorptive and angiogenic inhibitor profile

5. Indicate if the patient has already commenced therapy and duration

Dentist

1. Receive patient, evaluate modifiable risk factors, establish follow-up system

2. Before antiresorptive therapy:

- Conduct complete dental examination

- Perform necessary dental extractions and conservative dental and periodontal interventions

- Adjust prosthetics

- Educate the patient about the need for a lifelong daily commitment to oral care

- Encourage the correction of risk factors

3. During antiresorptive therapy:

- Encourage follow-up visits every 6 months

- Conduct complete dental examination

- Evaluate the oral status of oral soft and hard tissue

- Reinforce ongoing education about the importance of maintaining good oral hygiene

- Continue to reinforce modifiable risk factors

4. Follow patient's lesion status and report it to the oncologist

Dental specialist

1. Accept suspected patients

2. Management is determined by the stage, the severity of symptoms, functional impact and overall prognosis and should be on an 8-week follow-up schedule

3. Design a treatment plan and inform oncologist

4. Evaluate disease outcome

5. Make sure follow-up visits every 8 weeks

plan. ${ }^{74}$ For the patient, this would be a treatment loop of closed responsibility with all benefits directed to the patient. This proposed model represents a new paradigm for the management of MRONJ that is suitable for widespread promotion.

\section{Therapy for MRONJ}

From an in-depth study of MRONJ, conservative therapy is the mainstream treatment, ${ }^{75-77}$ as it can provide long-term relief. $^{9}$ According to the Clinical Practice Guidelines of the MASCC/ISOO/ ASCO, for patients with confirmed MRONJ, the treatment goal is to alleviate pain, control infection in soft and hard tissues, and decrease the progress or occurrence of osteonecrosis. ${ }^{4}$ This may be interpreted as the fact that the treatment for MRONJ has been clearly defined, delaying the progress of the disease with minimal cost to provide patients with a better after-treatment experience. Studies have reported that there is no significant difference in the rate of achieving a cure between surgical and nonsurgical treatments, ${ }^{78,79}$ with less aggressive surgical treatments producing better outcomes than those that are more aggressive. ${ }^{80}$ Therefore, in clinically asymptomatic conditions, conservative treatment would be the primary choice. New guidelines state that it is not recommended to intervene in asymptomatic bone exposure with aggressive surgical treatment. Prior to treatment, the multidisciplinary team should discuss thoroughly with the patient the risks and benefits of the proposed plan. ${ }^{4}$

Reported cases of successful treatments of MRONJ using teriparatide (TPTD) have verified that it is beneficial for osteoporotic patients with established MRONJ. TPTD has been used to treat MRONJ in animal studies, and positive outcomes have been observed. ${ }^{81}$ Successful treatment with TPTD for MRONJ has been found in several clinical case reports. ${ }^{82,83}$ Combination treatment with bone morphogenetic protein can enhance bone formation and promote bone regeneration. ${ }^{84}$ In patients with stage 3 MRONJ refractory to conservative management, weekly TPTD administration was shown to be as effective as daily TPTD in promoting bone healing and removing osteonecrotic tissue. ${ }^{85}$ However, no additional prospective randomised studies with convincing results of the treatment of MRONJ with TPTD have been published, so it is too early to support the use of TPTD as prevention or treatment for MRONJ. ${ }^{86}$

The combination of pentoxifylline and tocopherol has been used previously in the management of osteoradionecrosis, with significant improvement in symptomatology. ${ }^{87}$ Pentoxifylline is a nonselective phosphodiesterase inhibitor that displays good therapeutic results against osteoradionecrosis of the jaws and MRONJ. ${ }^{88-91}$ Tocopherol is a powerful scavenger of oxygen free radicals, which can reduce the damage caused by free radicals and necrosis. ${ }^{92}$ The study demonstrated that pentoxifylline and tocopherol are relatively inexpensive and simple to use and represent a safe and effective treatment for MRONJ. ${ }^{87}$ Studies have reported that pentoxifylline and tocopherol are potentially useful for the nonsurgical management of MRONJ. ${ }^{88,89}$ This treatment provides similar results towards healing as other nonsurgical treatment modalities with minimal side effects, costs and/or time burdens. Additional studies are required, however, to determine the optimal dosing and duration of treatment.

Antibiotics should not be abused at any stage of $\mathrm{MRONJ}^{93}$ because infection does not directly lead to its development. However, the majority of MRONJ patients have infection-related symptoms (stages 2 and 3). Therefore, at stages 2 and 3 with a substantial area of necrosis, antibacterial therapy contributes to the healing of MRONJ. When conservative treatment is unsatisfactory, surgical treatment is required. ${ }^{94,95}$ Surgical treatment modalities vary from marginal osteotomy to segmental osteotomy. Following the failure of conservative treatment, surgery is widely recommended in patients with hypoimmunity and reduced quality of life to avoid the risk of bacteraemia and septicaemia. ${ }^{96-}$ 98 It has been reported that weak patients with MRONJ who are unresponsive to conservative methods may have oral complications, including exposure to bone, infection, pain and discomfort, therefore requiring specialised care. ${ }^{99-101}$ Considering that these circumstances promote infection, it is necessary to perform surgery therapeutically. ${ }^{102}$ However, there is no clear guidance on how to determine the surgical plan for patients in stages 2 and 3 for whom conservative treatment was ineffective, so surgical treatment is therefore advocated. ${ }^{103-105}$ However, the type of 


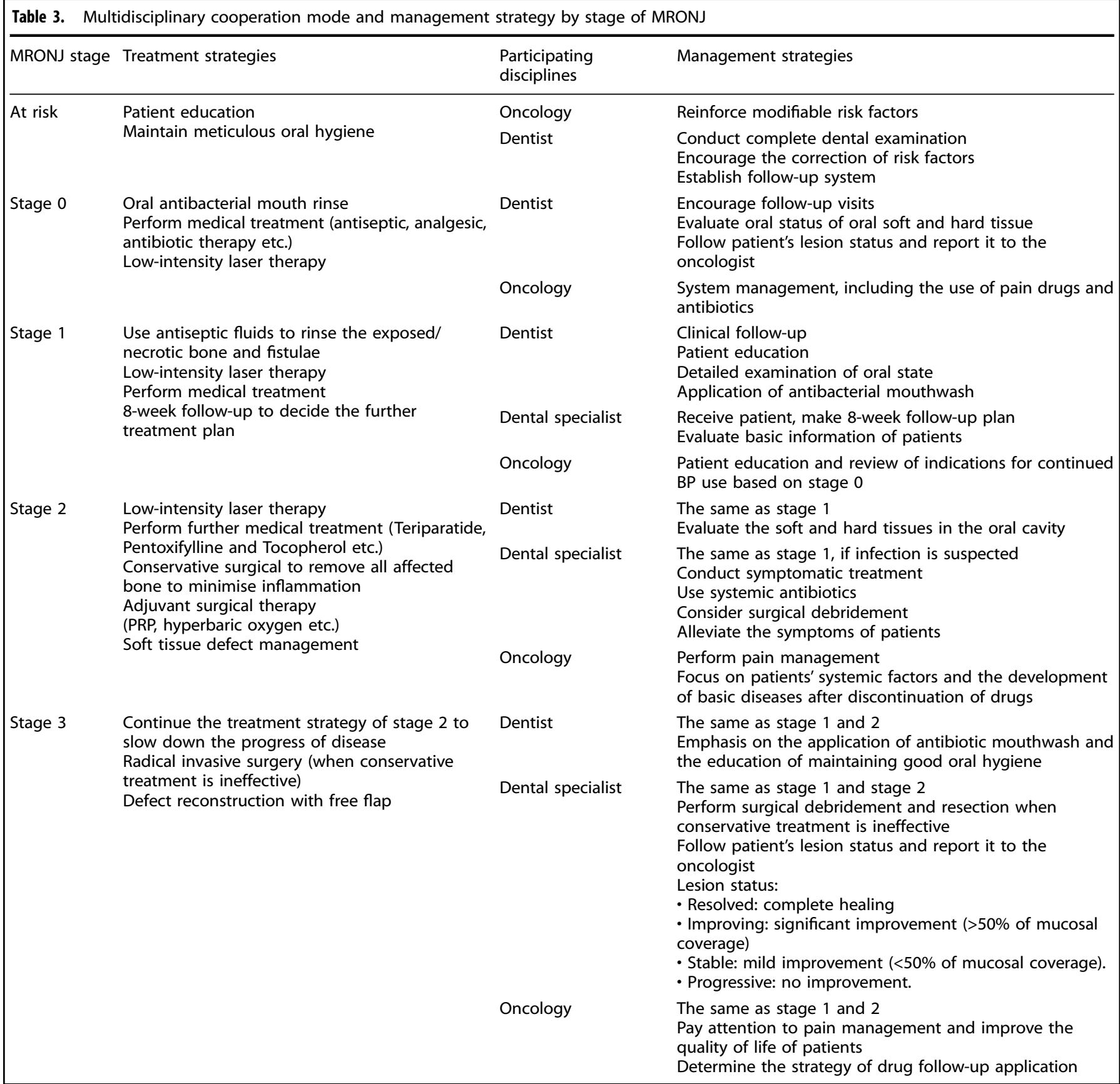

recommended surgical intervention, including preservation or segmental resection of the jaw, determination of the incisal margin of safety, soft tissue management and selection of functional reconstruction, is not clear.

Because the surgical treatment of MRONJ is difficult to quantify, expansion of the range of surgical resection is an important but uncertain factor for establishing the success of surgical treatment. ${ }^{103,106}$ The boundary for marginal osteotomy is based on the findings from the operation, such as bleeding and colour of the remaining bone, which are not always positively correlated with bone vitality. Inexperienced maxillofacial surgeons do not always find that performing marginal resection surgery is simple. It has been reported that bone resection could be performed under fluorescence guidance, and necrotic osteotomy selected during surgery could assist in distinguishing necrotic bone from healthy bone for standardising surgical treatment. ${ }^{107}$ Experienced maxillofacial surgeons are believed to have the capability to successfully treat affected areas using osteotomy.

There are also challenges in the restoration of soft and hard tissue function following the resection of a lesion. Due to soft tissue toxicity, unlike free flap reconstruction, buccal fat pad (BFP) flaps have been used to fill the dead space in necrotic bone resection. ${ }^{108,109}$ The main advantage of BFP flaps is their rich blood supply, flexibility, lack of age restrictions, safety and spontaneous formation of the epithelium. ${ }^{110,111}$ It has been reported that the use of BFP flaps makes it possible to close maxilla defects with two layers (BFP and mucoperiosteal flaps). ${ }^{12}$ Using this method, the majority of bone surfaces can be closed with BFP flaps, avoiding oral and nasal communication and infections of the maxillary sinus. Undoubtedly, this is the most 
appropriate treatment selection. Even if the BFP flap becomes exposed, spontaneous epithelialization occurs, which results in defect reconstruction.

Hyperbaric oxygen therapy is an effective adjuvant treatment for MRONJ. Hyperbaric oxygen can reduce inflammation and oedema and increase vasculogenesis, antimicrobial activity and tissue repair. ${ }^{113,114}$ It has been reported that hyperbaric oxygen alone cannot completely cure MRONJ. ${ }^{115}$ Therefore, it should be considered part of multimodal therapy. Hyperbaric oxygen is beneficial when used with antibiotics and surgery. ${ }^{116,117}$ Although current findings demonstrate that hyperbaric oxygen can improve MRONJ, the methodological limitations contribute to a lack of measurable effects. ${ }^{118,119}$ Therefore, the efficacy of hyperbaric oxygen therapy in MRONJ treatment requires further investigation.

Owing to the beneficial effects of low-intensity laser (LIL) treatment towards tissue healing, both alone and in combination with other treatment methods such as high-power lasers and surgical intervention, improved therapy can be achieved ${ }^{120-122}$ because LIL treatment can regulate metabolism, promote wound healing and relieve pain. ${ }^{123}$ It has been established that biological stimulation from LILs assists in healing both soft and hard tissues, especially in the treatment of early pathological changes. ${ }^{124,125}$ When combined with a high-power laser, a LIL is effective in inducing the complete healing of mucosa and reduction in microbial contamination. Studies have shown that surgery is an important treatment for patients with MRONJ, but when a LIL is used together with the removal of necrotic bone, better therapeutic effects can be obtained. ${ }^{123,124}$ Therefore, LIL has become an effective adjuvant therapy for MRONJ and is expected to become an alternative therapy. ${ }^{104}$

Platelet-rich plasma (PRP), an autologous source of growth factors, has been successfully used in bone regeneration and soft tissue healing. ${ }^{126,127}$ PRP can produce high concentrations of human platelets containing a variety of growth factors. In surgery for MRONJ, PRP appears to be particularly useful as an adjunct to surgical debridement and marginal osteotomy in patients with conservative treatment failure. ${ }^{128,129}$ However, there is no clear support for current PRP treatments, and more research is required to assess the potential of the treatment. ${ }^{130,131}$

Following an in-depth study of the disease, multiple therapeutic methods have been proposed, which can affect the outcome of the disease to a certain extent. There is no clear guidance on how to standardise the choice of treatment method from the multitude of effective alternatives. A sequential treatment pattern should be proposed, as shown in Table 3. The management and mode of treatment cooperation in multidisciplinary teams (MDTs) should be conducted during all periods of treatment. At all stages of disease progression, patient education should be emphasised and oral hygiene should be controlled by simple methods, such as oral antibacterial mouth rinse, which has an important role in inhibiting the development of the disease. LIL therapy is a possible choice for the treatment of osteonecrosis by aiding the reparative process. LILs can stimulate the growth of the vascular system and blood capillaries. ${ }^{123}$ At stage 0 in particular, the use of LIL therapy can control the progress of the disease, consistent with the treatment concept. At stage 1, the prevention of progressive infection is the key to blocking disease progression. The use of local antiseptic fluids to rinse the exposed/necrotic bone and fistulae and the application of LIL can provide a better local healing environment, promoting the prognosis of osteonecrosis. An 8-week follow-up examination should be performed, and a multidisciplinary consultation should determine the followup treatment plan based on the follow-up results. At stages 0 and 1 , the principal purpose of drug treatment is to prevent infection and control symptoms. When the disease progresses to stages 2 and 3 , drugs such as teriparatide, pentoxifylline and tocopherol can be used to delay the development of the disease, and the progress of infection can be controlled using antibiotics. When a drug or auxiliary LIL treatment for more than 2 weeks has no apparent effect, conservative surgery should be considered to remove all affected bone to minimise inflammation. At the same time, auxiliary treatment such as PRP or hyperbaric oxygen can be used to increase the success rate of surgery. ${ }^{104,132}$ After bone tissue infection has been controlled, an adjacent flap with abundant blood supply (such as a BFP or the modified submental island flap) should be first considered for the repair of soft tissue defects. ${ }^{133}$ In stage 3 , in case of ineffective conservative treatment, radical invasive surgery can be conducted to improve the quality of life of the patient. However, in order to reconstruct the dead space following the removal of damaged bone, free flap repair can be used to reconstruct soft tissue defects, which should be performed prior to correcting the hard tissue defect. From the summary of existing and effective treatment methods, it is hoped that standardised sequential therapy can be formulated as soon as possible.

\section{PROSPECT}

The pathogenesis of MRONJ has not been entirely clarified and may involve many factors within specific microenvironments. Multiple signalling pathways may be involved in the pathogenesis of MRONJ. At present, it is relatively certain that the TGF$\beta 1$ signalling pathway has a key role in the development of MRONJ, ${ }^{16,29,41}$ potentially representing a future research direction. Therefore, the study of the TGF- $\beta$ pathway and specific downstream sites may lead to a breakthrough in the pathogenesis of MRONJ. Although there are many studies on MRONJ, a consensus on a sequential treatment plan still requires additional research. If the accumulation of BPs in the jaw can be competitively inhibited while maintaining a drug-based treatment, this may be an effective method of preventing osteonecrosis of the jaw in the future. The multidisciplinary collaboration model of disease management should be affirmed. During treatment, each team member should integrate and share information about the treatment and perform his or her specified role at each stage, thereby preventing the occurrence or development of MRONJ to accomplish personalised treatment.

\section{ACKNOWLEDGEMENTS}

This study was supported by the National Natural Science Foundation of China (grant number: 81970924).

\section{ADDITIONAL INFORMATION}

Competing interests: The authors declare no competing interests.

\section{REFERENCES}

1. Marx, R. E. Pamidronate (Aredia) and zoledronate (Zometa) induced avascular necrosis of the jaws: a growing epidemic. J. Oral. Maxillofac. Surg. 61, 1115-1117 (2003).

2. Ruggiero, S. L. et al. American Association of Oral and Maxillofacial Surgeons position paper on medication-related osteonecrosis of the jaw-2014 update. American Association of Oral and Maxillofacial Surgeons. J. Oral. Maxillofac. Surg. 72, 1938-1956 (2014).

3. Dodson, T. B. The frequency of medication-related osteonecrosis of the jaw and its associated risk factors. Oral. Maxillofac. Surg. Clin. North Am. 27, 509-516 (2015).

4. Yarom, N. et al. Medication-related osteonecrosis of the jaw: MASCC/ISOO/ASCO clinical practice guideline. J. Clin. Oncol. 37, 2270-2290 (2019).

5. Lo, J. C. et al. Prevalence of osteonecrosis of the jaw in patients with oral bisphosphonate exposure. J. Oral. Maxillofac. Surg. 68, 243-253 (2010).

6. Nowicki, B. et al. Medication-related osteonecrosis of the jaw in a minipig model: parameters for developing a macroscopic, radiological, and microscopic grading scheme. J. Craniomaxillofac. Surg. 47, 1162-1169 (2019).

7. Khan, A. A. et al. Diagnosis and management of osteonecrosis of the jaw: a systematic review and international consensus. J. Bone Min. Res. 30, 3-23 (2015). 
8. Matsuura, T. et al. Distinct characteristics of mandibular bone collagen relative to long bone collagen: relevance to clinical dentistry. Biomed. Res. Int. 2014, 769414 (2014)

9. Saad, F. et al. Incidence, risk factors, and outcomes of osteonecrosis of the jaw: integrated analysis from three blinded active-controlled phase III trials in cancer patients with bone metastases. Ann. Oncol. 23, 1341-1347 (2012)

10. Yamaza, T. et al. Mouse mandible contains distinctive mesenchymal stem cells. J. Dent. Res. 90, 317-324 (2011).

11. Abdik, $\mathrm{H}$. et al. The effects of bisphosphonates on osteonecrosis of jaw bone: a stem cell perspective. Mol. Biol. Rep. 1, 763-776 (2019).

12. Taniguchi, N. et al. BPs induced reactive oxygen species inhibit proliferation and migration of oral fibroblast: a pathogenesis of bisphosphonate-related osteonecrosis of the jaw. J. Periodontol. https://doi.org/10.1002/JPER.19-0385 (2019).

13. Li, M. et al. Decreased osteogenic ability of periodontal ligament stem cells leading to impaired periodontal tissue repair in BRONJ patients. Stem Cells Dev. 29, 156-168 (2020).

14. de Souza Faloni, A. P. et al. Jaw and long bone marrows have a different osteoclastogenic potential. Calcif. Tissue Int. 88, 63-74 (2011).

15. Abe, T. et al. Zoledronic acid increases the circulating soluble RANKL level in mice, with a further increase in lymphocyte-derived soluble RANKL in zoledronic acid- and glucocorticoid-treated mice stimulated with bacterial lipopolysaccharide. Cytokine 83, 1-7 (2016).

16. Wehrhan, F. et al. Bisphosphonate-associated osteonecrosis of the jaw is linked to suppressed TGF $\beta 1$-signaling and increased Galectin-3 expression: a histological study on biopsies. J. Transl. Med. 9, 102 (2011).

17. Manzano-Moreno, F. J. et al. Bisphosphonate modulation of the gene expression of different markers involved in osteoblast physiology: possible implications in bisphosphonate-related osteonecrosis of the jaw. Int. J. Med. Sci. 15, 359-367 (2018)

18. Yasuda, H. et al. Identity of osteoclastogenesis inhibitory factor (OCIF) and osteoprotegerin (OPG): a mechanism by which OPG/OCIF inhibits osteoclastogenesis in vitro. Endocrinology 139, 1329-1337 (1998).

19. $\mathrm{Di}$, Nisio, C. et al. RANK/RANKL/OPG signaling pathways in necrotic jaw bone from bisphosphonate-treated subjects. Eur. J. Histochem. 59, 2455 (2015).

20. Chen, L. et al. Network-based method for identifying co-regeneration genes in bone, dentin, nerve and vessel tissues. Genes 10, E252 (2017).

21. Kim, H. J. et al. Zoledronate enhances osteocyte-mediated osteoclast differentiation by IL-6/RANKL axis. Int J. Mol. Sci. 20, E1467 (2019).

22. Shibahara, T. Antiresorptive agent-related osteonecrosis of the jaw (ARONJ): a twist of fate in the bone. Tohoku J. Exp. Med. 247, 75-86 (2019).

23. Hansen, T., Kunkel, M., Weber, A., James \& Kirkpatrick, C. Osteonecrosis of the jaws in patients treated with BPs-Histomorphologic analysis in comparison with infected osteoradionecrosis. J. Oral. Pathol. Med. 35, 155-160 (2006).

24. Di Fede, O. et al. The dental management of patients at risk of medicationrelated osteonecrosis of the jaw: new paradigm of primary prevention. Biomed. Res. Int. 2018, 2684924 (2018).

25. Soundia, A. et al. Zoledronate impairs socket healing after extraction of teeth with experimental periodontitis. J. Dent. Res. 97, 312-320 (2018).

26. Li, C. L. et al. Role of periodontal disease in bisphosphonate-related osteonecrosis of the jaws in ovariectomized rats. Clin. Oral. Implants Res. 27, 1-6 (2016).

27. Otto, S. et al. Bisphosphonate-related osteonecrosis of the jaw: is $\mathrm{pH}$ the missing part in the pathogenesis puzzle? J. Oral. Maxillofac. Surg. 68, 1158-1161 (2010).

28. Tsao, C. et al. Oral health risk factors for bisphosphonate-associated jaw osteonecrosis. J. Oral. Maxillofac. Surg. 71, 1360-1366 (2013).

29. Kim, S. et al. IL-36 induces bisphosphonate-related osteonecrosis of the jaw-like lesions in mice by inhibiting TGF- $\beta$-mediated collagen expression. J. Bone Min. Res. 32, 309-318 (2017).

30. Kalyan, S., Quabius, E. S., Wiltfang, J., Monig, H. \& Kabelitz, D. Can peripheral blood gammadelta T cells predict osteonecrosis of the jaw? An immunological perspective on the adverse drug effects of aminobisphosphonate therapy. $J$. Bone Min. Res. 28, 728-735 (2013).

31. Hagelauer, N., Pabst, A. M., Ziebart, T., Ulbrich, H. \& Walter, C. In vitro effects of bisphosphonates on chemotaxis, phagocytosis, and oxidative burst of neutrophil granulocytes. Clin. Oral. Investig. 19, 139-148 (2015).

32. Patntirapong, S. \& Poolgesorn, M. Alteration of macrophage viability, differentiation, and function by BPs. Oral. Dis. 24, 1294-1302 (2018).

33. Ferrari-Lacraz, S. \& Ferrari, S. Do RANKL inhibitors (denosumab) affect inflammation and immunity? Osteoporos. Int 22, 435-446 (2011).

34. Zhu, W. et al. Zoledronic acid promotes TLR-4-mediated M1 macrophage polarization in bisphosphonate-related osteonecrosis of the jaw. FASEB J. 33, 5208-5219 (2019).

35. Vincenzi, B. et al. Serum VEGF levels as predictive marker of bisphosphonaterelated osteonecrosis of the jaw. J. Hematol. Oncol. 5, 56 (2012).

36. Fantasia, J. E. The role of antiangiogenic therapy in the development of osteonecrosis of the jaw. Oral. Maxillofac. Surg. Clin. North Am. 27, 547-553 (2015).
37. Misso, G. et al. Evaluation of the in vitro and in vivo antiangiogenic effects of denosumab and zoledronic acid. Cancer Biol. Ther. 13, 1491-1500 (2012).

38. Kuroshima, S., Sasaki, M. \& Sawase, T. Medication-related osteonecrosis of the jaw: a literature review. J. Oral. Biosci. 61, 99-104 (2019).

39. Khan, A. A. et al. Case-based review of osteonecrosis of the jaw (ONJ) and application of the international recommendations for management from the International Task Force on ONJ. J. Clin. Densitom. 20, 8-24 (2017).

40. Tokuda, $\mathrm{H}$. et al. Involvement of MAP kinases in TGF-beta-stimulated vascular endothelial growth factor synthesis in osteoblasts. Arch. Biochem. Biophys. 415 117-125 (2003)

41. Lee, K. H. et al. Identifying genetic variants underlying medication-induced osteonecrosis of the jaw in cancer and osteoporosis: a case control study. J. Transl. Med. 17, 381 (2019).

42. Ishtiaq, S. et al. The effect of nitrogen containing bisphosphonates, zoledronate and alendronate, on the production of pro-angiogenic factors by osteoblastic cells. Cytokine 71, 154-160 (2015).

43. Reid, I. R., Bolland, M. J. \& Grey, A. B. Is bisphosphonate-associated osteonecrosis of the jaw caused by soft tissue toxicity? Bone 41, 318-320 (2007).

44. Simon, M. J., Niehoff, P., Kimmig, B., Wiltfang, J. \& Acil, Y. Expression profile and synthesis of different collagen types I, II, III, and V of human gingival fibroblasts, osteoblasts, and SaOS-2 cells after bisphosphonate treatment. Clin. Oral. Investig. 14, 51-58 (2010).

45. Border, W. A. et al. Natural inhibitor of transforming growth factor-beta protects against scarring in experimental kidney disease. Nature 360, 361-364 (1992).

46. Lescaille, G. et al. Osteonecrosis of the jaw and nonmalignant disease: is there an association with rheumatoid arthritis? J. Rheumatol. 40, 781-786 (2013).

47. de Molon, R. S. et al. Rheumatoid arthritis exacerbates the severity of osteonecrosis of the jaws (ONJ) in mice. A randomized, prospective, controlled animal study. J. Bone Min. Res. 8, 1596-1607 (2016).

48. Peer, A. \& Khamaisi, M. Diabetes as a risk factor for medication-related osteonecrosis of the jaw. J. Dent. Res. 94, 252-260 (2015).

49. Bastida-Lertxundi, N. et al. Pharmacogenomics in medication-related osteonecrosis of the jaw: a systematic literature review. Eur. Rev. Med. Pharm. Sci. 23, 10184-10194 (2019).

50. Katz, J. et al. Genetic polymorphisms and other risk factors associated with bisphosphonate induced osteonecrosis of the jaw. Int J. Oral. Maxillofac. Surg. 40, 605-611 (2011)

51. Borromeo, G. L. A large case-control study reveals a positive association between bisphosphonate use and delayed dental healing and osteonecrosis of the jaw. J. Bone Min. Res. 29, 1363-1368 (2014)

52. Qi, W. X., Tang, L. N., He, A. N., Yao, Y. \& Shen, Z. Risk of osteonecrosis of the jaw in cancer patients receiving denosumab: a meta-analysis of seven randomized controlled trials. Int J. Clin. Oncol. 19, 403-410 (2014).

53. Fedele, S. et al. Up to a quarter of patients with osteonecrosis of the jaw associated with antiresorptive agents remain undiagnosed. Br. J. Oral. Maxillofac. Surg. 53, 13-17 (2015).

54. Huber, F. A. et al. Medication-related osteonecrosis of the jaw-comparison of bone imaging using ultrashort echo-time magnetic resonance imaging and cone-beam computed tomography. Invest. Radio. 55, 160-167 (2020).

55. Sánchez-López, J. D., Cariati, P., Cambil-Martin, J., Villegas-Calvo, M. \& MorenoMartin, M. L. Use of bone scintigraphy in the early diagnosis of bisphosphonate related osteonecrosis of the jaw. Case report and review of the literature. J. Clin. Exp. Dent. 10, e1235-e1237 (2018).

56. Arce, K., Assael, L. A., Weissman, J. L. \& Markiewicz, M. R. Imaging findings in bisphosphonate-related osteonecrosis of jaws. J. Oral. Maxillofac. Surg. 67, 75-84 (2009).

57. Sim, le. W., Sanders, K. M., Borromeo, G. L., Seymour, J. F. \& Ebeling, P. R. Declining incidence of medication-related osteonecrosis of the jaw in patients with cancer. J. Clin. Endocrinol. Metab. 100, 3887-3893 (2015).

58. Filleul, O., Crompot, E. \& Saussez, S. Bisphosphonate-induced osteonecrosis of the jaw: a review of 2,400 patient cases. J. Cancer Res Clin. Oncol. 136, 1117-1124 (2010).

59. Kajizono, M. et al. Incidence and risk factors of osteonecrosis of the jaw in advanced cancer patients after treatment with zoledronic acid or denosumab: a retrospective cohort study. Biol. Pharm. Bull. 38, 1850-1855 (2015).

60. Hong, C. H. L. et al. A systematic review of dental disease management in cancer patients. Support Care Cancer 26, 155-174 (2018).

61. Vandone, A. M. et al. Impact of dental care in the prevention of bisphosphonateassociated osteonecrosis of the jaw: a single-center clinical experience. Ann. Oncol. 23, 193-200 (2012).

62. Stanton, D. C. \& Balasanian, E. Outcome of surgical management of bisphosphonate-related osteonecrosis of the jaws: review of 33 surgical cases. J. Oral. Maxillofac. Surg. 67, 943-950 (2009). 
63. Thumbigere-Math, V. et al. Bisphosphonate-related osteonecrosis of the jaw: clinical features, risk factors, management, and treatment outcomes of 26 patients. J. Oral. Maxillofac. Surg. 67, 1904-1913 (2009).

64. Yarom, N. et al. Rapid onset of osteonecrosis of the jaw in patients switching from bisphosphonates to denosumab. Oral. Surg. Oral. Med Oral. Pathol. Oral. Radio. 125, 27-30 (2018).

65. Rabelo, G. D. et al. Bisphosphonate-related osteonecrosis of the jaws and its array of manifestations. J. Maxillofac. Oral. Surg. 14, 699-705 (2015).

66. Clarke, B. M. et al. Bisphosphonates and jaw osteonecrosis: the UAMS experience. Otolaryngol. Head. Neck Surg. 136, 396-400 (2007).

67. Wessel, J. H., Dodson, T. B. \& Zavras, A. I. Zoledronate, smoking, and obesity are strong risk factors for osteonecrosis of the jaw: a case-control study. J. Oral. Maxillofac. Surg. 66, 625-631 (2008).

68. Carlson, E. R., Fleisher, K. E. \& Ruggiero, S. L. Metastatic cancer identified in osteonecrosis specimens of the jaws in patients receiving intravenous bisphosphonate medications. J. Oral. Maxillofac. Surg. 71, 2077-2086 (2013).

69. Basch, E. The missing voice of patients in drug-safety reporting. N. Engl. J. Med. 362, 865-869 (2010)

70. Gilligan, T. et al. Patient-clinician communication: American Society of Clinical Oncology consensus guideline. J. Clin. Oncol. 35, 3618-3632 (2017).

71. Schiodt, M. et al. A multicenter case registry study on medication-related osteonecrosis of the jaw in patients with advanced cancer. Support Care Cancer 26, 1905-1915 (2018)

72. Ferlito, S. et al. Treatment of bisphosphonate-related osteonecrosis of the jaws: presentation of a protocol and an observational longitudinal study of an Italian series of cases. Br. J. Oral. Maxillofac. Surg. 50, 425-429 (2012).

73. Rodrigues, P., Hering, F. \& Imperio, M. Safety of I.V. nonnitrogen bisphosphonates on the occurrence of osteonecrosis of the jaw: long-term follow-up on prostate cancer patients. Clin. Genitourin. Cancer 13, 199-203 (2015).

74. Lesclous, P. et al. Relevance of surgical management of patients affected by bisphosphonate-associated osteonecrosis of the jaws. A prospective clinical and radiological study. Clin. Oral. Investig. 18, 391-399 (2014).

75. Ortega, C. et al. Osteonecrosis of the jaw in prostate cancer patients with bone metastases treated with zoledronate: a retrospective analysis. Acta Oncol. 46, 664-668 (2007).

76. Hellstein, J. W. et al. Managing the care of patients receiving antiresorptive therapy for prevention and treatment of osteoporosis: executive summary of recommendations from the American Dental Association Council on Scientific Affairs. J. Am. Dent. Assoc. 142, 1243-12451 (2011).

77. Moretti, F., Pelliccioni, G. A., Montebugnoli, L. \& Marchetti, C. A prospective clinical trial for assessing the efficacy of a minimally invasive protocol in patients with bisphosphonate-associated osteonecrosis of the jaws. Oral. Surg. Oral. Med Oral. Pathol. Oral. Radio. Endod. 112, 777-782 (2011).

78. Thorn, J. J., Sorensen, H., Weis-Fogh, U. \& Andersen, M. Autologous fibrin glue with growth factors in reconstructive maxillofacial surgery. Int J. Oral. Maxillofac. Surg. 33, 95-100 (2004).

79. Scoletta, M., Arduino, P. G., Dalmasso, P., Broccoletti, R. \& Mozzati, M. Treatment outcomes in patients with bisphosphonate-related osteonecrosis of the jaws: a prospective study. Oral. Surg. Oral. Med Oral. Pathol. Oral. Radio. Endod. 110, 46-53 (2010).

80. Schubert, M. et al. The Saxon bisphosphonate register: therapy and prevention of bisphosphonate-related osteonecrosis of the jaws. Oral. Oncol. 48, 349-354 (2012).

81. Dayisoylu, E. H. et al. The effects of adjunctive parathyroid hormone injection on bisphosphonate-related osteonecrosis of the jaws: an animal study. Int J. Oral. Maxillofac. Surg. 42, 1475-1480 (2013).

82. Cheung, A. \& Seeman, E. Teriparatide therapy for alendronate-associated osteonecrosis of the jaw. N. Engl. J. Med. 363, 2473-2474 (2010).

83. El-Rabbany, M., Sgro, A., Lam, D. K., Shah, P. S. \& Azarpazhooh, A. Effectiveness of treatments for medication-related osteonecrosis of the jaw: a systematic review and meta-analysis. J. Am. Dent. Assoc. 148, 584-594 (2017).

84. Jung, J. et al. Short-term teriparatide and recombinant human bone morphogenetic protein-2 for regenerative approach to medication-related osteonecrosis of the jaw: a preliminary study. J. Bone Min. Res 32, 2445-2452 (2017).

85. Yoshiga, D. et al. Weekly teriparatide injections successfully treated advanced bisphosphonate-related osteonecrosis of the jaws. Osteoporos. Int. 24, 2365-2369 (2013).

86. Liu, Y. et al. Anabolic agents: what is beyond osteoporosis? Osteoporos. Int. 29, 1009-1022 (2018).

87. Martos-Fernandez, M. et al. Pentoxifylline, tocopherol, and clodronate for the treatment of mandibular osteoradionecrosis: a systematic review. Oral. Surg. Oral. Med Oral. Pathol. Oral. Radio. 125, 431-439 (2018).

88. Epstein, M. S., Wicknick, F. W., Epstein, J. B., Berenson, J. R. \& Gorsky, M. Management of bisphosphonate-associated osteonecrosis: pentoxifylline and tocopherol in addition to antimicrobial therapy. initial case Ser. Oral. Surg. Oral. Med Oral. Pathol. Oral. Radio. Endod. 110, 593-596 (2010).
89. Magremanne, M. \& Reychler, H. Pentoxifylline and tocopherol in the treatment of yearly zoledronic acid-related osteonecrosis of the jaw in a corticosteroidinduced osteoporosis. J. Oral. Maxillofac. Surg. 72, 334-337 (2014).

90. Owosho, A. A., Estilo, C. L., Huryn, J. M. \& Yom, S. K. Pentoxifylline and tocopherol in the management of cancer patients with medication-related osteonecrosis of the jaw: an observational retrospective study of initial case series. Oral. Surg. Oral. Med Oral. Pathol. Oral. Radiol. 122, 455-459 (2016).

91. Yalcin-Ulker, G. M., Cumbul, A., Duygu-Capar, G., Uslu, U. \& Sencift, K. Preventive effect of phosphodiesterase inhibitor pentoxifylline against medication-related osteonecrosis of the jaw: an animal study. J. Oral. Maxillofac. Surg. 75, 2354-2368 (2017).

92. Jiang, Q. Natural forms of vitamin E: metabolism, antioxidant, and antiinflammatory activities and their role in disease prevention and therapy. Free Radic. Biol. Med. 72, 76-90 (2014).

93. Ji, X. et al. Antibiotic effects on bacterial profile in osteonecrosis of the jaw. Oral. Dis. 18, 85-95 (2012).

94. Advisory Task Force on Bisphosphonate-Related Ostenonecrosis of the Jaws, American Association of Oral and Maxillofacial Surgeons. American association of oral and maxillofacial surgeons position paper on bisphosphonate-related osteonecrosis of the jaws. J. Oral. Maxillofac. Surg. 65, 369-376 (2007).

95. Ruggiero, S. L. Bisphosphonate-related osteonecrosis of the jaw: an overview. Ann. N. Y Acad. Sci. 1218, 38-46 (2011).

96. Bagán, J. et al. Recommendations for the prevention, diagnosis, and treatment of osteonecrosis of the jaw (ONJ) in cancer patients treated with bisphosphonates. Med Oral. Patol. Oral. Cir. Bucal 12, E336-E340 (2007).

97. Weitzman, R. et al. Critical review: updated recommendations for the prevention, diagnosis, and treatment of osteonecrosis of the jaw in cancer patientsMay 2006. Crit. Rev. Oncol. Hematol. 62, 148-152 (2007).

98. Lam, D. K., Sándor, G. K., Holmes, H. I., Evans, A. W. \& Clokie, C. M. A review of bisphosphonate-associated osteonecrosis of the jaws and its management. J. Can. Dent. Assoc. 73, 417-422 (2007).

99. Carter, G. D. \& Goss, N. A. N. Bisphosphonates and avascular necrosis of the jaws. Aust. Dent. J. 48, 268 (2003).

100. Pogrel, M. A. Bisphosphonates and bone necrosis. J. Oral. Maxillofac. Surg. 62, 391-392 (2004).

101. Ruggiero, S. L., Mehrotra, B., Rosenberg, T. J. \& Engroff, S. L. Osteonecrosis of the jaws associated with the use of bisphosphonates: a review of 63 cases. J. Oral. Maxillofac. Surg. 62, 527-534 (2004).

102. Ruggiero, S. L., Fantasia, J. \& Carlson, E. Bisphosphonate-related osteonecrosis of the jaw: background and guidelines for diagnosis, staging and management. Oral. Surg. Oral. Med Oral. Pathol. Oral. Radio. Endod. 102, 433-441 (2006).

103. Wilde, F. et al. The role of surgical therapy in the management of intravenous bisphosphonates-related osteonecrosis of the jaw. Oral. Surg. Oral. Med Oral. Pathol. Oral. Radio. Endod. 111, 153-163 (2011).

104. de Souza Tolentino, E. et al. Adjuvant therapies in the management of medication-related osteonecrosis of the jaws: systematic review. Head. Neck 41, 4209-4228 (2019).

105. McLeod, N. M., Patel, V., Kusanale, A., Rogers, S. N. \& Brennan, P. A. Bisphosphonate osteonecrosis of the jaw: a literature review of UK policies versus international policies on the management of bisphosphonate osteonecrosis of the jaw. Br. J. Oral. Maxillofac. Surg. 49, 335-342 (2011).

106. Seth, R., Futran, N. D., Alam, D. S. \& Knott, P. D. Outcomes of vascularized bone graft reconstruction of the mandible in bisphosphonate-related osteonecrosis of the jaws. Laryngoscope 120, 2165-2171 (2010).

107. Pautke, C. et al. Fluorescence-guided bone resection in bisphosphonate-related osteonecrosis of the jaws: first clinical results of a prospective pilot study. J. Oral. Maxillofac. Surg. 69, 84-91 (2011).

108. Berrone, M., Florindi, F. U., Carbone, V., Aldiano, C. \& Pentenero, M. Stage 3 medication-related osteonecrosis of the posterior maxilla: surgical treatment using a pedicled buccal fat pad flap: case reports. J. Oral. Maxillofac. Surg. 73, 2082-2086 (2015)

109. Melville, J. C. et al. A technique for the treatment of oral-antral fistulae resulting from medication-related osteonec-rosis of the maxilla: the combined buccal fat pad flap and radical sinusotomy. Oral. Surg. Oral. Med Oral. Pathol. Oral. Radio. 122, 287-291 (2016).

110. Mirancille, A. et al. Improvement of postnatal neovascularization by human adipose tissue-derived stem cells. Circulation 110, 349-355 (2004).

111. Farre-Guasch, E., Martí-Pages, C., Hernandez-Alfaro, F., Klein-Nulend, J. \& Casals, $\mathrm{N}$. Buccal fat pad, an oral access source of human adipose stem cells with potential for osteochondral tissue engineering: an in vitro study. Tissue Eng. Part C Methods 16, 1083-1094 (2010).

112. Hakobyan, K., Poghosyan, Y. \& Kasyan, A. The use of buccal fat pad in surgical treatment of 'Krokodil' drug-related osteonecrosis of maxilla. J. Craniomaxillofac. Surg. 46, 831-836 (2018). 
113. Lee, N. K. et al. A crucial role for reactive oxygen species in RANKL-induced osteoclast differentiation. Blood 106, 852-859 (2005).

114. Freiberger, J. J. Utility of hyperbaric oxygen in treatment of bisphosphonaterelated osteonecrosis of the jaws. J. Oral. Maxillofac. Surg. 67, 96-106 (2009)

115. Rupel, K. et al. A systematic review of therapeutical approaches in bisphosphonates-related osteonecrosis of the jaw (BRONJ). Oral. Oncol. 50, 1049-1057 (2014).

116. Freiberger, J. J. et al. Hyperbaric oxygen treatment and bisphosphonate-induced osteonecrosis of the jaw: a case series. J. Oral. Maxillofac. Surg. 65, 1321-1327 (2007).

117. Freiberger, J. J. et al. What is the role of hyperbaric oxygen in the management of bisphosphonate-related osteonecrosis of the jaw: a randomized controlled trial of hyperbaric oxygen as an adjunct to surgery and antibiotics. J. Oral. Maxillofac. Surg. 70, 1573-1583 (2012).

118. Rollason, V. et al. Interventions for treating bisphosphonate-related osteonecrosis of the jaw (BRONJ). Cochrane Database Syst. Rev. 2, CD008455 (2016).

119. Beth-Tasdogan, N. H., Mayer, B., Hussein, H. \& Zolk, O. Interventions for managing medication-related osteonecrosis of the jaw. Cochrane Database Syst. Ver. 10, CD012432 (2017).

120. Favia, G., Tempesta, A., Limongelli, L., Crincoli, V. \& Maiorano, E. Medicationrelated osteonecrosis of the jaw: surgical or non-surgical treatment? Oral. Dis. 24, 238-242 (2018).

121. Altay, M. A., Tasar, F., Tosun, E. \& Kan, B. Low-level laser therapy supported surgical treatment of bisphosphonate related osteonecrosis of jaws: a retrospective analysis of 11 cases. Photomed. Laser Surg. 32, 468-475 (2014).

122. Vescovi, P. et al. Nd:YAG laser biostimulation in the treatment of bisphosphonate-associated osteonecrosis of the jaw: clinical experience in 28 cases. Photomed. Laser Surg. 26, 37-46 (2008).

123. Vescovi, P. et al. Surgical approach and laser applications in BRONJ osteoporotic and cancer patients. J. Osteoporos. 2012, 585434 (2012).

124. Scoletta, M., Arduino, P. G., Reggio, L., Dalmasso, P. \& Mozzati, M. Effect of low-level laser irradiation on bisphosphonate-induced osteonecrosis of the jaws: preliminary results of a prospective study. Photomed. Laser Surg. 28, 179-184 (2010).

125. Vescovi, P. et al. Early surgical laser-assisted management of bisphosphonaterelated osteonecrosis of the jaws (BRONJ): a retrospective analysis of 101 treated sites with long-term follow-up. Photomed. Laser Surg. 30, 5-13 (2012).

126. Wang, J. V., Schoenberg, E., Saedi, N. \& Ibrahim, O. Platelet-rich plasma, collagen peptides, and stem cells for cutaneous rejuvenation. J. Clin. Aesthet. Dermatol. 13, 44-49 (2020).
127. Plachokova, A. S., Nikolidakis, D., Mulder, J., Jansen, J. A. \& Creugers, N. H. Effect of plateletrich plasma on bone regeneration in dentistry: a systematic review. Clin. Oral. Implants Res. 19, 539-545 (2008).

128. Curi, M. M. et al. Bisphosphonate-related osteonecrosis of the jaws-an initial case series report of treatment combining partial bone resection and autologous platelet-rich plasma. J. Oral. Maxillofac. Surg. 69, 2465-2472 (2011).

129. Adornato, M. C., Morcos, I. \& Rozanski, J. The treatment of bisphosphonateassociated osteonecrosis of the jaws with bone resection and autologous platelet-derived growth factors. J. Am. Dent. Assoc. 138, 971-977 (2007).

130. Lopez-Jornet, P., Sanchez Perez, A., Amaral Mendes, R. \& Tobias, A. Medicationrelated osteonecrosis of the jaw: is autologous platelet concentrate application effective for prevention and treatment? A systematic review. J. Craniomaxillofac. Surg. 44, 1067-1072 (2016).

131. Del Fabbro, M., Gallesio, G. \& Mozzati, M. Autologous platelet concentrates for bisphosphonate-related osteonecrosis of the jaw treatment and prevention. A systematic review of the literature. Eur. J. Cancer 51, 62-74 (2015).

132. Bennardo, F. et al. Autologous platelet-rich fibrin injections in the management of facial cutaneous sinus tracts secondary to medication-related osteonecrosis of the jaw. Dermatol. Ther. 26, e13334 (2020).

133. Myoken, Y., Fujita, Y. \& Okamoto, T. Modified submental island flap for the surgical treatment of 4 patients with stage 3 medication-related osteonecrosis of the mandible. J. Oral. Maxillofac. Surg. 78, 29-34 (2020).

(c) Open Access This article is licensed under a Creative Commons Attribution 4.0 International License, which permits use, sharing adaptation, distribution and reproduction in any medium or format, as long as you give appropriate credit to the original author(s) and the source, provide a link to the Creative Commons license, and indicate if changes were made. The images or other third party material in this article are included in the article's Creative Commons license, unless indicated otherwise in a credit line to the material. If material is not included in the article's Creative Commons license and your intended use is not permitted by statutory regulation or exceeds the permitted use, you will need to obtain permission directly from the copyright holder. To view a copy of this license, visit http://creativecommons. org/licenses/by/4.0/.

(c) The Author(s) 2020 